\title{
Jacques HAMEL
}

sociologue, département de sociologie, Université de Montréal

(1989)

\section{"Pour la méthode de cas. Considérations méthodologiques et perspectives générales”}

\author{
Un document produit en version numérique par Jean-Marie Tremblay, bénévole, \\ professeur de sociologie au Cégep de Chicoutimi \\ Courriel: jean-marie tremblay@uqac.ca \\ Site web pédagogique : http://www.uqac.ca/jmt-sociologue/ \\ Dans le cadre de: "Les classiques des sciences sociales" \\ Une bibliothèque numérique fondée et dirigée par Jean-Marie Tremblay, \\ professeur de sociologie au Cégep de Chicoutimi \\ Site web: http://classiques.uqac.ca/ \\ Une collection développée en collaboration avec la Bibliothèque \\ Paul-Émile-Boulet de l'Université du Québec à Chicoutimi \\ Site web: http://bibliotheque.uqac.ca/
}


Cette édition électronique a été réalisée par Jean-Marie Tremblay, bénévole, professeur de sociologie au Cégep de Chicoutimi à partir de :

\section{Jacques Hamel}

"Pour la méthode de cas. Considérations méthodologiques et perspectives générales”.

Un article publié dans la revue Anthropologie et Sociétés, vol. 13 no 3, 1989, pp. 59-72. Numéro intitulé : "Méthodologies et univers de recherche”. Québec: Département d'anthropologie, Université Laval.

M. Jacques Hamel est sociologue au département de sociologie de l'Université de Montréal.

[Autorisation formelle réitérée le 16 juin 2004 par l'auteur, M. Hamel, de diffuser cet article]

Q.

Courriel : jacques.hamel@umontreal.ca

Polices de caractères utilisée :

Pour le texte: Times New Roman, 14 points.

Pour les citations : Times New Roman, 12 points.

Pour les notes de bas de page : Times New Roman, 12 points.

Édition électronique réalisée avec le traitement de textes Microsoft Word 2004 pour Macintosh.

Mise en page sur papier format : LETTRE (US letter), 8.5’’ x 11'’)

Édition numérique réalisée le 9 juillet 2007 à Chicoutimi, Ville de Saguenay, province de Québec, Canada. 
Jacques Hamel

\section{"Pour la méthode de cas. Considérations méthodologiques et perspectives générales"}

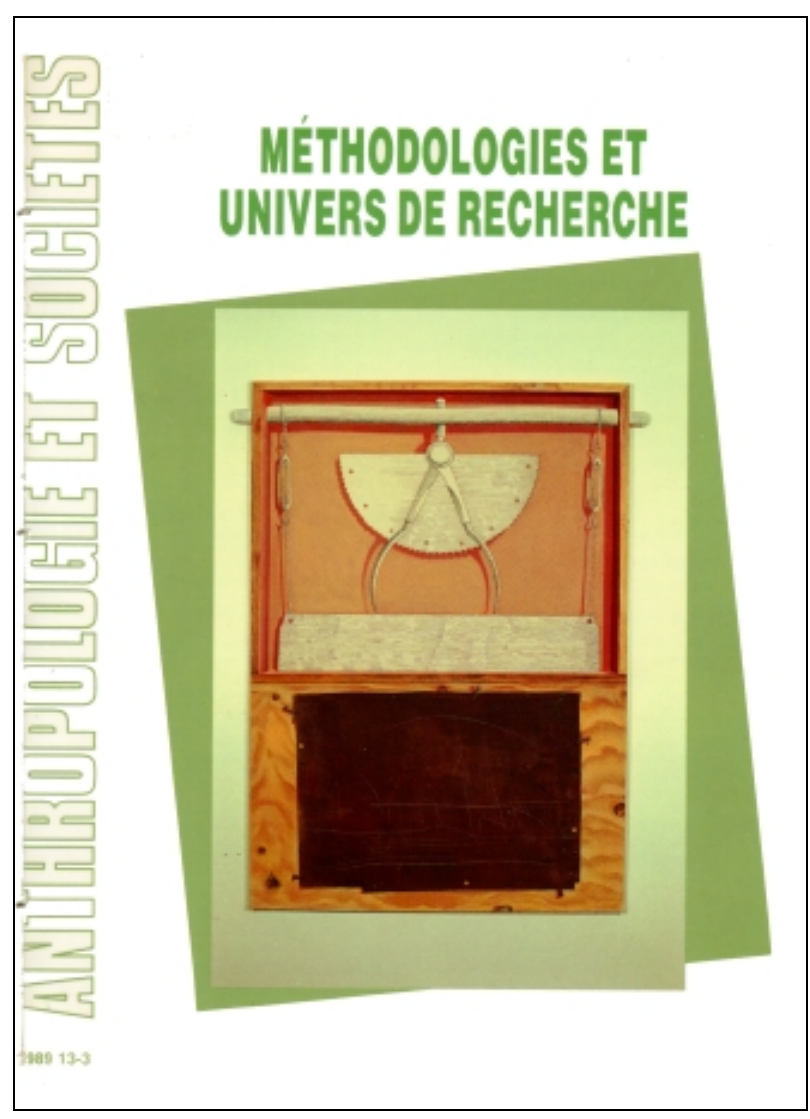

Un article publié dans la revue Anthropologie et Sociétés, vol. 13 no 3, 1989, pp. 59-72. Numéro intitulé : "Méthodologies et univers de recherche”. Québec : Département d'anthropologie, Université Laval. 


\section{Table des matières}

$\underline{\text { Introduction }}$

Le conflit des méthodes

La question de la représentativité

Le rapport du local au global

La valeur heuristique de la méthode de cas

$\underline{\text { Remarques finales en guise de conclusion }}$

$\underline{\text { Références }}$

Résumé / Abstract 
Jacques Hamel

“Pour la méthode de cas. Considérations méthodologiques

et perspectives générales".

Un article publié dans la revue Anthropologie et Sociétés, vol. 13 no 3, 1989, pp. 59-72. Numéro intitulé : "Méthodologies et univers de recherche”. Québec : Département d'anthropologie, Université Laval.

\section{Introduction}

La méthode de cas connaît actuellement un regain de faveur en sciences sociales après un fort recul. Ce retour en force s'est engagé sous l'impulsion du procès des méthodes statistiques, qui a fait surgir de multiples oppositions paraissant aujourd'hui trop évidentes pour être discutées : quantitatif/qualitatif ; objectivité/subjectivité ; généralité/particularité ; macro/micro-sociologie ; représentativité/exemplarité ; et maintes autres alimentées par des disputes et querelles ${ }^{1}$. Ces débats ont conféré un caractère d'irréductibilité à des oppositions expressément fondées sur la spécificité de leurs termes. Nées de conflits de méthodes dont la dimension politique a été naguère - et à bon droit - soulignée ${ }^{2}$, ces dernières ont acquis une forme canoni-

1 Trop souvent, le débat s'est confiné dans d'âpres disputes et controverses sur le statut des méthodes quantitatives, qu'on désirait épurer de toute dimension qualitative. Sur les conflits, notamment politiques, autour des méthodes et du statut conféré aux démarches qualitatives et quantitatives, voir le remarquable article d'A. Pires (1982) ; voir également Cicourel et Knorr-Cetina (1981).

2 Point n'est besoin de rappeler le conflit des méthodes qu'on a pris l'habitude de camper entre l'université Colombia et l'Université de Chicago et dont on a sur- 
que à laquelle il est difficile de se soustraire. Sans vouloir raviver la querelle, l'on peut rapporter cet extrait d'entrevue où Maurice Godelier précise les attributs et vertus de la méthode anthropologique qui se résume, à ses yeux, à l'observation participante, propre à l'analyse de cas particuliers et fondant la spécificité de l'anthropologie :

[...] l'unité de ce domaine vient de la méthode qu'on emploie. Cette méthode est l'observation participante. C'est-à-dire l'immersion prolongée dans les rapports sociaux locaux, la descente dans le puits. Et c'est à partir des informations recueillies par un observateur au sein d'un petit groupe social que se construisent les théories de l'anthropologie.

Gwenael 1985 : 148

Selon Godelier, la reconstitution empirique de rapports sociaux observés dans une configuration locale permet d'atteindre à la « logique sociale d'ensemble ». Cette méthode appartiendrait de plein droit aux dispositifs d'expérimentation en laboratoire des sciences de la nature, dont la rigueur fait tant envie. Ces qualités reconnues sont, pourtant, déliées de toute visée de généralité dans la suite de l'entretien, comme si la méthode de cas était, d'entrée de jeu, rebelle à la perspective macroscopique :

L'anthropologie est un instrument puissant, mais qui a ses limites, puisque c'est à partir de ses observations personnelles sur un petit nombre d'individus que l'anthropologue essaie de reconstruire une logique sociale d'ensemble. Qu'un anthropologue observe par exemple les rapports sociaux dans une usine, il aura besoin de connaître la place de cette usine dans l'économie régionale et nationale.

Gwenael 1985 : 149

Cet entretien n'apporte rien de nouveau : la méthode de cas est définie comme une première approche destinée à la mise au jour d'une spécificité, d'une particularité d'ordre qualitatif, que l'approche macroscopique devrait obligatoirement mettre en perspective sur un plan

tout retenu les querelles proprement politiques, voire les conflits de personnalité. 
global, comme si cette dernière imposait son patronage. Il est dès lors acquis que la méthode de cas ne peut, d'aucune façon, atteindre à la généralité et sans doute, inversement, les méthodes statistiques ne peuvent mettre en lumière la particularité. Ce point de vue dresse des barrières étanches entre approches et méthodes, qui ne font que conforter les dichotomies déjà mentionnées. Ces méthodes peuvent certes être complémentaires, mais suivant un ordre de préséance rigide : la méthode de cas devra précéder l'approche macroscopique, la première se justifiant au plan méthodologique par l'exposition d'une singularité que les méthodes statistiques mettront à l'épreuve en vue d'une généralisation. Sans vouloir contester cette complémentarité méthodologique, sans nul doute fondée, il sera montré dans ce texte que la méthode de cas possède une valeur macroscopique, qui lui était autrefois reconnue, mais qu'on lui dénie maintenant en raison de conflits de méthode où la revendication de représentativité n'est posée - et ne reste posée - que suivant une logique purement statistique.

\section{Le conflit des méthodes}

\section{$\underline{\text { Retour à la table des matières }}$}

La méthode de cas - voire le qualitatif - serait donc spécifique à la saisie du particulier, du singulier, de la mise au jour de l'individu social et de ses actions, mobilisées à son échelle, tandis que le quantitatif serait propre à la saisie du collectif, du structurel et même du social. Le triomphe de la quantité a été proclamé en vertu des valeurs explicatives et prédictives, du point de vue du général, attribuées aux méthodes statistiques et auxquelles la description monographique ne saurait prétendre ; à tout le moins qu'on ne saurait lui reconnaître en raison de la petite taille de ses unités d'analyse. Par petite taille de l'unité d'analyse, on entend surtout des populations limitées et géographiquement restreintes et non la qualité sociologique présumée de ces 
unités. C'est sur ce point précis que les discussions ont achoppé et, pour cette raison, il convient de le mettre en relief.

Les premières enquêtes ethnographiques ont principalement porté sur de petites sociétés, au sein desquelles les acteurs sociaux sont en situation d'interaction directe, définissant des communautés réelles, c'est-à-dire dans le cadre de milieux écologiques et géographiques proprement déterminés, et où les rapports extérieurs ont fort peu d'importance ${ }^{3}$. Les descriptions monographiques ont d'abord été tirées des récits de voyage des missionnaires avant de donner naissance à l'anthropologie et à la sociologie des sociétés traditionnelles ou rurales. Sans que cela n'ait été dûment explicité dans ces oeuvres pionnières ni n'ait fait ensuite l'objet d'attentions ou de considérations méthodologiques, ces descriptions minutieuses avaient une valeur de généralité de par les vertus « naturelles », pourrait-on dire, des unités d'analyse reconstituées, de par les propriétés des rapports sociaux constitutifs de ces petites sociétés. Elles permettaient d'atteindre à la spécificité d'une société en raison des vertus méthodologiques conférées à chacun des cas privilégiés. Suivant le langage en vigueur dans ce type d'anthropologie et de sociologie des sociétés traditionnelles, aujourd'hui fortement décriées et trop souvent à tort, la description monographique permettait de révéler les " arêtes d'une société », à partir de cas certes particuliers, un village, une tribu, mais, de par les propriétés spécifiques des rapports sociaux constitutifs de ces sociétés, présentant néanmoins une portée macroscopique.

Chaque cas constituait donc une espèce de découpage méthodologique propice à atteindre ce qui caractérisait une société déterminée dans sa généralité. L'analyse monographique de cas autorisait dans pareilles conditions, relatives - il importe de le noter - à la nature sociologique de ces sociétés, des explications de valeur générale. La par-

3 L'anthropologie a fourni une quantité considérable d'études remarquables dont l'énumération serait trop longue et fort périlleuse. Qu'il nous soit permis néanmoins de signaler l'ouvrage de Maurice Godelier ( 1982). 
ticularité mise au jour avait ainsi une valeur de généralité puisque ces cas constituaient, de par les qualités de ce découpage spécifique, une sorte de prisme permettant d'expliquer une société dans ce qui la caractérise. Il y aurait sans nul doute un rapprochement à opérer entre un cas défini à la manière d'un prisme et le foyer défini dans les sciences optiques, c'est-à-dire le point d'un continuum où la densité acquise de la convergence de rayons lumineux permet d'apprécier les propriétés de la lumière. Les qualités de découpage renvoyaient donc aux propriétés spécifiques des rapports sociaux constitutifs de ces sociétés et, au surplus, ce découpage se confondait avec des attributs géographiques, écologiques et politiques déterminant directement le cas retenu sous forme d'une entité proprement physique, géographique, politique, etc. Dans les premiers travaux monographiques, les vertus méthodologiques reconnues au cas, acquises d'entrée de jeu, n'exigeaient aucune explication ${ }^{4}$.

L'entrée de ces sociétés dans la modernité (l'expression est utilisée ici pour sa commodité et son économie) s'est opérée par l'éclatement de cette nature sociologique (interaction directe et restriction des échanges extérieurs à l'univers social, politique, religieux et cosmique) sous l'impulsion de la découverte des nouveaux continents, de la sécularisation des croyances et des institutions, de la création de moyens de communication modernes et de la généralisation des échanges économiques. Dans pareil contexte, la nature de ces sociétés se différencie dans ses fondements mêmes, brisant le caractère monolithique d'autrefois, au sein de rapports sociaux économiques, politiques et idéologiques désormais spécifiques. L'organisation de ces sociétés est à rechercher dans une totalité organique déterminée du point de vue

4 Il n'en est cependant pas ainsi des premières monographies faites au Québec par des anthropologues américains, tels E. Hughes, H. Miner, etc. Il faut lire la remarquable préface de l'édition originale (1939) de Saint-Denis : un village québécois et l'introduction où Miner expose les visées de son étude et les critères l'ayant conduit au choix de ce village, qui ne relèvent en rien du hasard ou d'une vision folklorique (Miner 1985). 
de cette différenciation sociale et non plus de celui d'un strict espace géographique, écologique ou politique 5 .

Les ethnologues furent, dès lors, aux prises avec le problème des limites de la monographie locale dont la valeur présumée de généralité était compromise en raison de la différenciation des rapports sociaux où la vie sociale - dans l'ordre de l'organisation d'une société - n'est en rien réductible à un espace géographique ou politique mais renvoie, par exemple, aux impératifs de la production et des échanges économiques.

Le village - voire la tribu d'aujourd'hui - n'est donc plus le terrain d'élection de la mise au jour d'une spécificité, reconstituée avec soin et donnant lieu à une explication dont la valeur de généralité était naguère parfaitement autorisée. En limitant la définition de leur objet, de leur unité d'analyse, à des unités géographiques ou politiques, les anthropologues n'ont fait que reprendre un découpage déjà donné, ne parvenant pas ainsi à tirer de leçon des œuvres pionnières dont l'objet - la définition du cas -, reposant certes sur des frontières politiques ou géographiques, était néanmoins, dans le même temps, de par la nature de ces sociétés, un découpage à valeur méthodologique aux fins de l'explication visée dans sa généralité.

La formulation la meilleure de ce type de structure villageoise [visée par les anthropologues], écrit Clifford Geertz, est peut-être de la représenter en recourant au modèle de l'intersection de plans d'organisation sociale théoriquement distincts [...] Un village n'est pas un hameau ni un groupe fréquentant une même église, mais un exemple concret d'intersection de différents plans d'organisation sociale, dans un lieu défini de manière large.

Geertz, cité dans Champagne 1982 : 11

L'emphase accordée à la description monographique de villages, sans aucune considération méthodologique, donnait prise à bon droit à

5 Il convient ici d'utiliser le terme, fort peu commode, de « déterritorialisation » de la vie sociale ; voir Rosanvallon (1979 : 99 et passim). 
des reproches d'absence de représentativité. Il ne pouvait donc plus être question de faire crédit d'une valeur de généralité, ne serait-ce que virtuelle, à la monographie locale puisque la mise au jour de particularités ne pouvait revendiquer pareil statut en raison du choix du cas, privilégié selon des motifs étrangers à une véritable stratégie méthodologique de recherche « d'intersection des plans de l'organisation sociale $»$.

Le déclin de la valeur de la monographie, de la méthode de cas, fut au surplus accentué par la recherche permanente d'une qualité exotique des cas privilégiés, ne faisant que doubler les reproches d'absence de représentativité. Cette dernière fut dès lors strictement réservée aux méthodes statistiques en plein essor. La recherche d'une spécificité, d'une particularité par étude de cas pouvant être posée comme explicative apparaît de cette façon inappropriée à une démarche où la quantité de cas est propice à sa mise en lumière et, en vertu des qualités reconnues au nombre, confortée du même coup dans une généralité qui ne fait pas de doute.

Les objectifs de la méthode de cas et des méthodes quantitatives ne s'opposent pas. La première est destinée à l'exposition d'une spécificité, posée comme explicative, dont la valeur de généralité est acquise de par les qualités du cas en tant qu'« intersection de différents plans de l'organisation sociale », relatives à une stratégie théorique et méthodologique qui autorise cette valeur. Les méthodes quantitatives ont pareille visée, mais la spécificité dégagée en termes explicatifs acquiert d'emblée une valeur de généralité en raison de la quantité de cas considérés permettant sans contredit la découverte présumée de l'« intersection des plans de l'organisation sociale » à laquelle il est alors conféré un statut de représentativité. 


\section{La question de la représentativité}

\section{$\underline{\text { Retour à la table des matières }}$}

La représentativité serait donc, à première vue, le point faible de la méthode de cas, des enquêtes monographiques. L'emphase mise sur la détermination de la représentativité a contribué à la fortune de la qualité comme mesure définissant l'explication. L'opposition de la qualité et de la quantité dans la détermination de la représentativité pouvait ainsi être étendue à la définition proprement dite de toute mesure constitutive de l'explication où il apparaît que, strictement, le nombre peut remplir ce double office. Cette opposition se situe donc sur deux plans où, ainsi qu'il sera montré, le nombre n'est cependant point requis de façon obligée ou, dans l'usage qu'il en est fait, n'altère en rien le caractère qualitatif de la représentativité et de la mesure définissant l'explication.

La mesure elle-même est la recherche de l'explication. Elle n'est [a priori] ni qualitative ni quantitative, elle est la recherche des propriétés de l'objet défini dont la construction théorique constitue l'explication. Elle serait qualitative au sens second, i.e. de l'ordre d'une interprétation - sociologique - des qualités de l'objet.

Houle 1979 : 5

La mesure n'a pas à susciter une opposition, mais bel et bien un rapport qualité-quantité pour donner lieu à l'objectivation d'une expérience au sein d'un savoir théorique. La quantité peut à cette fin, de l'avis de G. Granger, remplir un double office : le dénombrement et la mesure, entendue au sens de la saisie des propriétés d'un objet.

Dans le dénombrement, la quantité n'intervient que comme nombre entier naturel, et ne se trouve avoir avec l'objet qu'une relation tout à fait extrin- 
sèque. Ce sont divers exemplaires de cet objet qui sont énumérés et non pas l'objet lui-même qui se trouve intrinsèquement associé à un nombre.

Granger $1988: 5$

Il en est autrement de la mesure comme saisie des propriétés d'un objet, « qui consiste à faire correspondre à des opérations portant sur l'objet et ses parties un système d'êtres mathématiques, qui sont originairement des nombres, mais qu'on peut concevoir de manière beaucoup plus générale »(ibidem). Le nombre peut donc être mis à contribution soit en tant qu'outil de récurrence soit aux fins de la définition d'index opératoires. Il convient de situer les deux usages potentiels du nombre sur des terrains distincts en vue d'en établir sans ambiguïté le statut. Si la définition du nombre en tant qu'outil de récurrence ne pose pas de problème, au sens de la réduction virtuelle des qualités d'un objet à un calcul, il en est tout autrement de sa contribution à la constitution d'index opératoires où " l'accent est mis sur une correspondance entre un système d'opérations concrètes portant sur l'objet et les propriétés formelles d'un système de grandeurs " (idem : 108). Or, à cette fin, le nombre peut être mis en vedette ou ne tenir qu'un rôle vicariant tellement cette spécification fonctionnelle, certes exprimable en termes mathématiques, n'est point en elle-même quantitative et réductible à un calcul mathématique.

La saisie des propriétés d'un objet, objectif affiché de l'explication, peut être définie en résumé, comme travail, au sens de G. Granger d'une opposition de forme à contenu dont l'institution « est toujours, et à tous les degrés d'élaboration, le premier moment décisif de l'objectivation d'une expérience, de sa transposition dans un système symbolique. Nous proposons même de reconnaître cet acte d'institution sous les figures proto-symboliques qu'elle revêt lorsqu'une forme est physiquement imposée à un phénomène matériel, et nous pensons y découvrir le sens le plus fondamental et le plus général d'un travail »(1987 : 202). L'opposition de la quantité et de la qualité, trop aisément entendue comme irréductible, ne serait en fait que conditions et modes de 
saisie distincts de ce rapport de forme à contenu dont il n'a été donné du premier qu'un exposé succinct, mais où il a été néanmoins remarqué que la saisie qualitative est maintes fois dissimulée dans le mode de saisie donnant lieu à la quantité.

Le rapport du contenu à une forme, c'est-à-dire la réduction des qualités, des propriétés d'un objet à une forme, peut certes être opéré par voie métrique, souvent commode et féconde, mais peut très bien être fixé par le langage, qui autorise un calcul si on entend par ce mot « l'idée d'opérations explicitement et univoquement définies et réglées » (Granger 1988 : 13). La forme sera ici conceptualisée par la réduction des qualités d'un objet à une structure de deux façons différentes, le dénombrement et l'index opératoire, « l'une privilégiant les éléments objets, l'autre les opérations, distinction domin[ant] les techniques de description dont l'étude détaillée serait à la base d'une épistémologie de la connaissance scientifique qualitative » (idem : 109). Le rapport du contenu à une forme peut dès lors se manifester de différentes façons, complémentaires, présenter les formes acquises de la réduction de la qualité que $\mathrm{G}$. Granger définit, en premier lieu, comme « repérage dans un réseau de référence [où] la forme sera décrite par la désignation d'éléments distingués » (ibidem). La saisie qualitative sera « donnée par une information - au sens de la théorie de l'information - sur la forme ». (idem : 110).

On entend [ici] la mise en évidence d'un tout et son découpage en parties.

L'instrument de cette opération de repérage est un canevas, ou réseau abstrait, convenablement adapté à la mise en place des fragments du puzzle et à la reconstitution du tout au moyen de ses parties.

Granger 1988 : 117

Le second mode de présentation de la forme est l'établissement d'un groupe de transformations assurant une compréhension, dans la mesure où « ramener la saisie d'une forme à celle de l'invariant d'un 
groupe de transformations, c'est en effet faire mieux en un sens que la décrire : on met alors à nu le secret ressort de toute pensée objective, qui est de jouer sur la réciprocité de la constitution d'un objet et du fonctionnement d'un système opératoire dont il serait le support en même temps que le produit. Ainsi la forme n'apparaît-elle plus comme une donnée brute, et l'explication de cette réciprocité est certainement l'un des sens les plus caractéristiques que l'on puisse donner au mot comprendre (idem : 112) ${ }^{6}$. La compréhension, dès qu'elle acquiert un degré de rigueur, se mue déjà en explication au moyen de la considération des singularités, selon le sens donné à l'expression singularité par le mathématicien R. Thom dans sa fameuse théorie des catastrophes : «Une singularité peut toujours être considérée comme provenant d'un espace régulier $E$ par concentration en un point d'une figure globale plongée dans cet espace E » (idem : 114).

Par ce mot d'explication nous entendons ici le rapport du local au global, sur lequel le mathématicien R. Thom a si justement insisté. On a vu qu'il caractérise la singularité comme « concentration » du global dans le local. Mais il suggère plus généralement que la démarche théorique s'oppose à la démarche pragmatique comme la visée de problèmes globaux, à résoudre par réduction à des situations locales typiques, s'oppose à la visée de problèmes locaux, à résoudre par des moyens globaux ... Il reconnaît dans la science elle-même cette double orientation. Mais l'intelligence des formes telle qu'il l'entend, aspirant comme on l'a noté à la saisie de leur engendrement, supposerait essentiellement le mouvement qui est celui de la connaissance qualitative par excellence et qui part des singularités pour y déceler et y redéployer le global.

Granger $1988: 114$

La saisie qualitative des propriétés d'un objet peut donc donner lieu à une description, une compréhension et une explication et ainsi être

6 «Comprendre suppose assurément déjà quelque description suffisamment adéquate et précise, fût-elle sommaire. Mais on ne comprend la forme que si l'on établit les relations et les contraintes qui associent les parties en lesquelles la description a découpé le tout. Un découpage qui serait seulement descriptif pourrait bien être tout à fait arbitraire ; un découpage compréhensif exige que des relations apparaissent entre les « morceaux » (Granger, 1988 : 117). 
soit une étape préparatoire soit une étape finale, puisque l'explication est ici reconnue possible dans sa valeur de généralité en vertu d'une concentration du global dans le local déterminant la nature structurale de cette mesure. Celle-ci est donc perfectionnée et se recommande d'une rigueur fort éloignée de ce caractère vague, subjectif, imaginaire, singulier au mauvais sens du mot, hautement proclamé par des partisans de l'approche qualitative mal éclairés, comme s'il était besoin de rajouter au peu de crédit accordé à son statut et à sa valeur,

Cette rigueur est fondée dans l'ordre de la description, de la compréhension et de l'explication des propriétés empiriques d'un objet, où le recours au nombre - faut-il encore le répéter - n'est ni obligé ni interdit, définissant une mesure qualitative de portée macroscopique suivant la mise en lumière de la nature structurale de leur singularité, caractérisée par le dessein descriptif et compréhensif de toute explication. Il est donc permis de penser qu'il existe une sorte de préséance méthodologique dans la détermination de la mesure - quantitative et qualitative - suivant laquelle l'explication ne peut s'échafauder que sur la base d'une description des propriétés d'un objet ${ }^{7}$. C'est sous ce même jour qu'il faut considérer la fameuse question de la représentativité où la méthode de cas retrouve dès lors des lettres de noblesse perdues depuis longue date tant la « représentativité statistique » a préva-

7 Gilles Granger, avec son pessimisme coutumier à l'égard de la conquête possible du caractère scientifique des sciences humaines, remarque : « Dans les sciences de l'homme, où ni la quantification ni une connaissance qualitative véritablement objectivante ne semblent avoir encore atteint leur maturité, il se peut même qu'il soit vain de trop espérer de la première, et que l'avenir soit à une espèce qualitative du savoir. Mais une telle connaissance supposerait que soient authentiquement constitués comme objets les phénomènes qu'étudient ces sciences, et qu'aucune confusion ne soit plus possible entre la qualité vécue de la saisie immédiate, et la qualité pensée et manipulée comme forme » (Granger 1988 : 120). À l'instar de G. Houle (1986), nous pensons que le passage de la qualité vécue à la qualité pensée est justement assuré par la description ici entendue - comme d'ailleurs dans la suite du présent texte - dans un sens extensif, incluant la compréhension au sens de G. Granger. Voir aussi Quéré et al. (1985). 
lu sous une forme canonique impérieuse aujourd'hui décriée, souvent pour de mauvaises raisons.

L'approche monographique offre seule la possibilité de préciser les conditions sociologiquement pertinentes de la représentativité, puisque s'attardant à décrire les processus concrets de la formation des usages sociaux ou de l'évolution des institutions, elle met à jour les facteurs les plus importants, les moments de rupture les plus déterminants du moins pour chaque culture ou chaque objet d'étude.

Zonabend $1985: 358$

La monographie, la méthode de cas ${ }^{9}$, ne renvoie donc pas à un âge de pierre de l'histoire des sciences de la société. Par la description, elle est le passage obligé de la mesure des propriétés d'un objet, et le dénombrement trouvera ses assises par la saisie qualitative de cette mesure. Il n'est rien ici de vraiment neuf : l'histoire des sciences, selon Granger, a très bien montré que les théories explicatives dans les sciences pures ont été précédées de théories descriptives sur lesquelles

8 F. Zonabend ajoute : « Mais aucun ethnologue poursuivant ce type de recherche ne revendique la représentativité, au sens statistique du terme, de l'unité sociale étudiée. Il prend certes soin de définir le cadre de référence dans lequel il oeuvre, de montrer la pertinence sociale de l'objet qu'il construit, mais les groupes sociaux sur lesquels porte son enquête - du fait de leur faible ampleur démographique -apparaissent toujours spécifiques. Mais spécifiques au regard de quoi ? Les grandes enquêtes à échantillonnage statistique ne permettent guère de répondre » $(1985: 35)$.

9 Le rapprochement fait ici entre "monographie » et «méthode de cas » ou " étude de cas » n'est en rien gratuit et ne doit pas entraîner de confusion. Le terme «monographie »est ici entendu au sens de l'Écoie de Chicago, de la tradition des monographies de E.C. Hughes; non pas au sens des «monographies villageoises » d'une certaine ethnologie française - aujourd'hui fortement mise en question (voir Bromberger 1987, Zonabend 1985) - qui mettent strictement en évidence la valeur exotique des cas observés (ce qui est décrié dans cet article). Parce que l'accent est mis sur cet exotisme, recherché en priorité, ces études monographiques de village ne recèlent ainsi aucune qualité comparative, contrairement à la tradition monographique de l'École de Chicago où la comparaison est constitutive de l'approche monographique, où la méthode comparative est directement mobilisée dans le travail monographique proprement dit. 
elles ont pu s'échafauder avec un degré accru de rigueur, ce qui rend caduques les oppositions quantité/qualité, local/global, singularité/généralité. On peut tirer profit de ces enseignements et résumer la spécificité des méthodes des sciences de la société par l'expression « positivisme tempéré », assurément plus féconde que le vain dualisme objectivité/subjectivité.

\section{Le rapport du local au global}

$\underline{\text { Retour à la table des matières }}$

L'étude des pratiques et stratégies économiques francophones au Québec offre un exemple de ce passage du local au global. L'économie francophone a été définie de façon classique comme dominée, subordonnée à une économie anglophone, d'abord anglo-saxonne puis américaine ${ }^{10}$. Mais de quoi est faite cette économie, que peuvent ou pouvaient être les pratiques économiques francophones dans le cadre de cette domination ? Ces questions ne sont pas de peu d'intérêt, car si ce caractère de domination a été amplement souligné dans des travaux qui ont fait date, la nature de l'économie francophone n'a guère été considérée, si ce n'est dans des enquêtes de terrain auxquelles on accorde peu de crédit ${ }^{11}$. Il s'agissait donc de découvrir la spécificité de

10 Voir particulièrement Saint-Germain (1973) ; aussi Savaria (1975). Pour un tableau général, voir Sales (1985) et Paquet (1985).

11 Voir les remarques fort sévères qu'A. Sales adresse aux études de cas, notamment à celle de Norman W. Taylor : «L'intérêt de [l'analyse d'A. Raynauld] est d'abord d'être représentative de l'ensemble de la population. Elle évite donc de tomber dans le travers des études de cas non suivies d'analyses plus extensives et qui, malgré tout, prétendent à un discours généralisant, comme par exemple la fameuse thèse, pourtant méthodologique ment biaisée, de Taylor » (1985 : 343). Voir également la réplique de Gérard Bélanger : « Même chez les universitaires des sciences sociales, les grandes fresques imprécises m'apparaissent mieux appréciées que les travaux plus sobres et plus fouillés sur des questions précises. Les critères des universitaires ne se différencieraient pas de ceux qui sont utilisés par les mass media » (1985 : 361). 
cette économie francophone qu'on avait peine, au départ, à déterminer puisque c'est sa sujétion qui fut en permanence mise au premier plan. En première approche, il ne pouvait être question de tirer un échantillon de pratiques représentatives de cette économie à cause de l'absence de définition qualitative, c'est-à-dire de la description de ses propriétés empiriques. L'analyse, dans cette perspective, s'est amorcée à partir d'études de cas, Les pratiques économiques constitutives de l'entreprise Forano de Plessisville ont été privilégiées en raison de l'histoire du développement de cette région, qui n'a pas été touchée par les capitaux étrangers, contrairement à la majorité des régions du Québec ${ }^{12}$, et dont l'industrialisation se résume à l'établissement de la Forano. Ce cas constitue donc un observatoire privilégié pour l'analyse de la spécificité des pratiques économiques francophones en vertu des propriétés sociologiques du milieu social considéré. La spécificité du développement de celui-ci confère au cas une valeur de représentativité suivant la visée de l'étude : l'analyse de l'économie francophone par le biais d'une forme présumée spécifique. La reconstitution empirique des pratiques économiques constitutives de cette entreprise, à l'aide de données et d'observations relevant de la description monographique, permet ainsi d'atteindre à cette spécificité, à la nature particulière et originale des pratiques économiques francophones, dont la valeur de représentativité attribuée est confortée par les attributs sociologiques du milieu social considéré, relatifs à des processus sociaux qui ont déterminé l'histoire de la société québécoise. Ces qualités, ces vertus heuristiques pourrions-nous dire, ne sont aucunement attribuées de façon péremptoire, mais sont déterminées par une stratégie inductive définissant ce cas dans le cadre d'un découpage méthodologique propice au passage du local au global. Parce qu'il n'est point permis ici de définir de prime abord ses pratiques économiques comme proprement inféodées à des capitaux étrangers, ce cas, certes particulier, révèle néanmoins des propriétés spécifiques de l'économie francophone qui ne peuvent et ne doivent en rien être réduites à une domination ou une dépendance directe. La valeur de cette spécificité

12 Voir Hughes (1972). 
peut être définie comme générale, c'est-à-dire caractéristique de cette économie, en raison des vertus attribuées au cas par l'explication méthodologique du découpage qui a justifié son choix. Cette spécificité est dévoilée, à travers ce cas particulier, dans des conditions de laboratoire, par la distinction entre les pratiques économiques qui en sont constitutives et celles d'autres cas où ces pratiques, certes aussi francophones, seraient subsumées dans un capitalisme étranger dont les effets définissent justement la domination ou la dépendance qui occupe le premier plan de l'explication. Celle-ci ne peut être réduite au caractère de subordination de l'économie francophone puisque si pareil rapport existe, c'est qu'il y a une économie dominante et une économie dominée. La spécificité de cette dernière doit être recherchée et mise en jeu aux fins de l'explication de ce rapport de domination.

\section{La valeur heuristique de la méthode de cas}

$\underline{\text { Retour à la table des matières }}$

Cet exemple montre assez les vertus de la méthode de cas et son recours obligé dans la recherche d'une spécificité - pour ce qui nous concerne, de la nature spécifique de rapports sociaux -définissant une saisie qualitative qui se recommande sans aucun doute de l'explication. La valeur de la spécificité mise au jour mérite le nom de représentativité au sens d'attributs propres à un objet, qui permettent de l'expliquer. Dans notre exemple, la reconstitution monographique a montré que les pratiques économiques constitutives du cas de la Forano étaient faites de rapports de parenté et d'alliance 13 dont la prégnance - à des degrés divers - est dès lors présumée générale. Cette hypothèse vaut jusqu'à preuve du contraire, c'est-à-dire la découverte

13 Du point de vue du recrutement de la main-d'œuvre, de l'organisation du travail, de la division du travail, de la mobilisation du capital. Voir Hamel, Houle et Sabourin (1984). 
de pratiques économiques proprement francophones dont la spécificité invaliderait la valeur représentative du cas de la Forano. La validité de l'hypothèse peut être poussée à un plus haut degré par la multiplication et la comparaison de cas, mais cette démarche prospective apportera de meilleurs résultats si l'hypothèse est affinée grâce à la mise en relief des particularités constitutives des autres cas.

Cette confrontation proprement heuristique permet au surplus de saisir la nature spécifique des rapports sociaux révélée dans son mouvement, étant entendu que " toute réalité sociale doit être considérée comme le résultat d'un procès, résultat qui est lui-même en mouvement et ne prend forme qu'au sein et au terme d'un processus qui lui confère ses caractères, ses propriétés spécifiques » (Godelier 1983 : 378). C'est pourquoi l'expression "à des degrés divers » employée plus haut a été soulignée. Car l'économie francophone peut être expliquée dans sa tradition, par la confrontation de cas, par l'observation d'une différenciation des rapports de parenté, constitutifs des stratégies et pratiques économiques francophones, donnant lieu à des rapports proprement économiques dont la primauté est, à tous égards, l'expression des transformations de cette économie. Le cas de la Forano a été ainsi compare a celui de la fonderie Gosselin, située à Drummondville, où le capitalisme étranger a pris racine avec une telle force que l'économie francophone n'a pu que se subordonner à ses exigences et contraintes. La comparaison ne pouvait donc que mettre en lumière des pratiques économiques francophones fortement différenciées en raison de la présence d'un capitalisme étranger dans un milieu défini par le titre français de la monographie de Hughes (1972) comme la « rencontre de deux mondes ». Cette présence a d'abord déterminé les transformations de cette économie francophone, puis de la société québécoise dans sa totalité, suivant le titre anglais, qui ne saurait tromper : French Canada in Transition.

L'étude de la fonderie Gosselin vient donc mettre en lumière la spécificité des pratiques économiques constitutives du cas de la Forano, par la mise au jour de particularités, relatives à la nature sociolo- 
gique différenciée du milieu drummondvillois, propices à l'observation de ces pratiques économiques francophones dans leur mouvement, c'est-à-dire leurs transformations. Il s'agit enfin de définir cellesci dans le cadre d'une théorie de la transition ${ }^{14}$ aux fins d'une explication dont la valeur de généralité ne fait pas de doute puisqu'elle est étayée par l'étude d'un autre cas dont la spécificité s'avère en parfait accord avec la stratégie méthodologique préconisée.

\section{Remarques finales en guise de conclusion}

$\underline{\text { Retour à la table des matières }}$

Parce qu'elle permet la mise au jour de particularités, de singularités constitutives de cas dûment privilégiés, la méthode de cas était la voie requise pour l'explication de J'économie francophone dans d'autres termes qu'en tant qu'économie dominée. La valeur de ces cas détermine un découpage méthodologique autorisant la généralité, méthodologique et non pas statistique, de l'explication. L'hypothèse théorique ainsi dégagée peut fort bien être mise à l'épreuve par l'augmentation du nombre de cas. Cette démarche est donc progressive et, sous le patronage des premières études de cas, sont mises enjeu, de proche en proche, une rigueur proprement opératoire et la consolidation d'une hypothèse posée, jusqu'à preuve du contraire, comme explicative.

14 Voir les travaux récents de Maurice Godelier (1981, 1987a, 1987b). 


\section{Références}

$\underline{\text { Retour à la table des matières }}$

ALGER C.F. (éd.)

1988 «Interconnexions entre le local et le mondial », Revue internationale des sciences sociales, 117 : 359-484.

BÉLANGER G.

1985 «La lecture des économistes est-elle si pauvre ? ", Recherches Sociographiques, XXVI, 3 : 361-364.

BROMBERGER C.

1987 « Du grand au petit. Variations des échelles et des objets d'analyse dans l'histoire récente de l'ethnologie de la France » : 67-94, in I. Chiva et U. Jeggle (éd.), Ethnologies en miroir. Paris : Éditions de la Maison des Sciences de l'Homme.

CASTELLS M.

1970 «Les nouvelles frontières de la méthodologie sociologique », Information sur les sciences sociales, 9, 6 : 79-108.

CHAMPAGNE P.

1982 "Statistique, monographie et groupes sociaux » : 3-16, in Études dédiées à Madeleine Grawitz. Genève : Dalloz.

CHAPOULIE J.M. 
1984 «Everett C. Hughes et le développement du travail de terrain en sociologie », Revue française de sociologie, XXV : 582-608.

1985 «Préface » : 9-22, in H.S. Becker, Outsiders. Paris : Éditions A.-M. Métaillé.

CICOUREL A.V. et K. Knorr-Cetina

1981 Advances in Social Theory and Methodology; Toward an Integration of Micro- and Macro-Sociologies. Boston, London : Routledge \& Kegan Paul.

CIPRIANI R.

1987 «Quantité et qualité dans l'analyse sociologique : faux dilemme ou débat idéologique dans la connaissance scientifique » 25-37, in J. Wodz (éd.), Problèmes de la sociologie qualitative. Katowice Université de Silésie.

COLLINS R.

1981a «Micro-translation as a Theory-building Strategy » : 81108, in A.V. Cicourel et K. Knorr-Cetina (éd.).

1981b « On the Microfoundations of Macrosociology », American Journal of Sociology, 86, 5 : 984-1014.

CORNAERT M. et C. Saint-Blancat

1988 «Le local et la contemporanéité : interférence du micro et macrosociologique », Espaces et Sociétés, 4-5 (n.s.) : 277291. 


\section{DECOSTER $M$.}

1985 "Lois, modèles et déterminismes sociologiques », Revue de l'Institut de sociologie, 1-2 : 191-206.

DION M.

1974 «Des monographies en sociologie » : 91-129, in M. Jollivet (éd.), Les collectivités rurales françaises. Tome 2 : Sociétés paysannes ou luttes de classes ou village ? Paris : Armand Colin.

GEERTZ C.

1986 Savoir local, savoir global. Paris : Presses Universitaires de France.

\section{GIDDENS A.}

1988 La constitution de la société. Paris : Presses Universitaires de France.

GINZBURG C.

1982 «Préface » : 7-29, in Le fromage et les vers. Paris : Flammarion.

\section{GODELIER M.}

1981 «D'un mode de production à l'autre : théorie de la transition », Recherches sociologiques, XII, 2 : 161-193.

1982 La production des Grands Hommes. Paris : Fayard.

1983 «Formation économique et sociale » : 377-380, in G. Labica (éd.), Dictionnaire critique du marxisme. Paris: Presses Universitaires de France. 
1987a «L'analyse des processus de transition », Information sur les sciences sociales, 26, 2 265-293.

1987b "Introduction l'analyse des processus de transition », Revue internationale des sciences sociales, 114 : 501-512.

GRANGER G.-G.

1987 «Contenus formels et dualité », Manuscrito, X, 2 : 195-210.

1988 Pour la connaissance philosophique. Paris : Odile Jacob.

GWENAEL A.

1985 «Entretien avec Maurice Godelier »: 147-156, in Rencontres avec le monde. Paris : La Découverte.

HAMEL J., G. Houle et P. Sabourin

1984 «Stratégies économiques et développement industriel: l'émergence de Forano », Recherches sociographiques, XXV, 2 : 189-209.

HOULE G.

1979 "Présentation », Sociologie et Sociétés, XIV, 1 : 3-6.

1986 «Histoires et récits de vie : la redécouverte obligée du sens commun » : 35-51, in D. Desmarais et P. Grell (éd.), Les récits de vie. Montréal : Éditions Saint-Martin.

HUGHES E.C.

1972 Rencontre de deux mondes. Montréal : Boréal Express.

JACOB P. (éd.) 
1980 De Vienne à Cambridge. L'héritage du positivisme logique. Paris : Gallimard.

MIGUELEZ R.

1977 La comparaison interculturelle. Montréal : Les Presses de l'Université de Montréal. [Livre en préparation pour Les Classiques des sciences sociales. JMT.]

1984 «L'articulation du général et du particulier : une approche méthodologique dans le champ des sciences sociales », Philosophiques, XI, 2 : 251-275.

MINER H.

1985 Saint-Denis : un village québécois. Montréal : Hurtubise $\mathrm{HMH}$.

PAQUET G.

1985 « Le fruit dont l'ombre est la saveur » : réflexions aventureuses sur la pensée économique au Québec », Recherches sociographiques, XXVI, 3 : 364-397.

PIRES A.

1982 «La méthode qualitative en Amérique du Nord : un débat manqué (1918-1960) », Sociologie et Sociétés, XIV, 1 : 1529.

QUÉRÉ L. et al.

1985 Décrire : un impératif' ? Description, explication, interprétation en sciences sociales. Paris : Éditions de l'École des Hautes Études en Sciences Sociales. 
ROSANVALLON P.

1979 Le capitalisme utopique. Paris : Éditions du Seuil.

SAINT-GERMAIN M.

1973 Une économie à libérer. Montréal : Les Presses de l'Université de Montréal.

SALES A. (avec la collaboration de L. Dumais)

1985 «La construction sociale de l'économie québécoise », Recherches sociographiques, XXVI, 3 : 319-360.

SAVARIA J.

1975 «Le Québec est-il une société périphérique ? », Sociologie et Sociétés, VII, 2: 115-127. [Texte disponible dans Les Classiques des sciences sociales. JMT.]

VOVELLE M.

1985 «De la biographie à l'étude de cas », Sources. Travaux et histoire, 3-4 : 191-198.

YIN R.K.

1985 Case Study Research. Design and Methods. Beverly Hills, London : Sage Publications.

ZONABEND F.

1985 «Du texte au prétexte. La monographie dans le domaine européen », Études rurales, 87-88 : 33-38. 


\section{RÉSUMÉ/ABSTRACT}

\section{Pour la méthode de cas \\ Considérations méthodologiques et perspectives générales}

Retour à la table des matières

Cet article vise à poser dans des termes neufs et féconds la valeur de l'approche monographique, de l'étude de cas, qui connaît aujourd'hui un regain de faveur dans les sciences sociales. Il est montré que la monographie ne peut être aucunement limitée à l'approche microscopique, mais qu'elle permet bien d'atteindre à la généralité. Ce point de vue est explicité par des considérations sur l'histoire de la monographie, dans le développement de l'anthropologie particulièrement, mais aussi par des considérations d'ordre épistémologique et méthodologique déterminant ce qui est ici appelé le passage du local au global, dont une recherche empirique sur l'économie francophone au Québec apporte un exemple.

\section{For the Case Study Method Methodological Considerations and General Perspectives}

This paper reassesses in new terms the value of the case study method - the monographic approach - which is regaining the favor of social scientists. The author shows that the monography cannot be confined to a microscopic view, but does permit generalization. This position is elaborated by some considerations on the history of monography, notably within the development of anthropology, but also by some epistemological and methodological considerations about the transition from local to global settings. The results of an empirical study about the french-speaking economy in Québec illustrate it. 
Jacques Hamel

Département de sociologie

Université de Montréal

C.P. 6128, succursale A

Montréal (Québec)

Canada H3C 3J7 